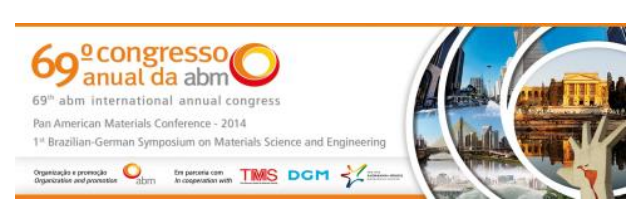

Tema: Metalurgia física e comportamento de materiais em temperaturas elevadas

\title{
ESTUDO DA INTERAÇÃO ENTRE DENSIDADE DE LINHAS DE CAMPO E PRESENÇA DE FASE SIGMA DE UM AÇO INOXIDÁVEL DUPLEX ATRAVÉS DE DO ESTUDO DE MEDIDAS DE TENSÃO HALL*
}

\section{Resumo}

\author{
Edgard de Macedo Silva ${ }^{1}$ \\ Ana Lucia Silva Souza Andrade ${ }^{2}$ \\ Bruno Ribeiro de Araújo ${ }^{3}$ \\ Josinaldo Pereira Leite 4 \\ Walter de Macêdo Lins Fialho \\ João Pereira Leiter ${ }^{5}$
}

Aços inoxidáveis duplex apresentam boas propriedades mecânicas e de resistência à corrosão, mas quando são colocados para trabalhar em altas temperaturas como no processo de soldagem e de tratamento térmico ocorre o surgimento de fases que comprometem as suas propriedades. Dentre elas, a fase sigma $(\sigma)$ é a que apresenta um maior efeito de fragilização por ser rica em cromo e empobrecer a matriz desse elemento, bem como por apresentar dureza em torno de 1000HV. No presente trabalho, uma metodologia não destrutiva, baseada na interação entre densidade de linhas de campo e um aço inoxidável duplex, é apresentada para o acompanhamento da formação da fase $\sigma$ na temperatura de $800^{\circ} \mathrm{C}$ em um aço envelhecido por até 2 horas. Diferentes intensidades de campo foram geradas por um eletroímã e o fluxo de linhas de campo foi aplicado no material de estudo. A densidade de linhas de campo resultante foi medida por um sensor de efeito Hall. Os resultados foram correlacionados com resultados de microscopia eletrônica de varredura (MEV), dureza e difração de raios-X (DRX) e mostraram que os valores de tensão hall reduziram à medida que a fração volumétrica da fase $\sigma$ aumentou. $A$ formação dessa fase não magnética influenciou no sinal obtido pelo sensor Hall. Existiu uma correlação entre a densidade de linhas de campo resultante e a presença da fase fragilizante, apontando para uma metodologia capaz de acompanhar a formação dessa fase.

Palavras-chave: Fase sigma; Ensaio não destrutivo; Densidade de linhas de campo.

\section{STUDY OF THE INTERACTION BETWEEN DENSITY OF LINES AND FIELD PRESENCE OF SIGMA PHASE OF A DUPLEX STAINLESS STEEL THROUGH THE STUDY HALL VOLTAGE MEASUREMENT}

\begin{abstract} the formation of this phase.

Keywords: Sigma phase; Nondestructive test; Magnetic flux density.

Engenharia Mecânica Doutor, professor, mecânica, IFPB, PB, Brasil.

Automação Industrial, Tecnólogo, Estudante, Automação, IFPB, PB, Brasil.

Engenharia Elétrica, graduando, Estudante, Elétrica, IFPB, PB, Brasil.

Engenharia Mecânica Doutor, professor, mecânica, UFPB, PB, Brasil.

Engenharia Mecânica Mestre, professor, mecânica, IFPB, PB, Brasil.
\end{abstract}

Duplex stainless steels have good mechanical and corrosion resistance properties, but when submitted to high temperatures as in welding and heat treatment process occurs the formation of phases that compromise their properties. Among then, the sigma phase $(\sigma)$ is what causes the most embrittlement effect to be rich in chromium and reduce the matrix of this element, as well as presenting hardness around $1000 \mathrm{HV}$. In this study, a non-destructive methodology, based on the interaction between magnetic flux density and a duplex stainless steel it is parsed for monitoring the formation of $\sigma$ phase at a temperature of $800^{\circ} \mathrm{C}$, for samples aged for times up to 2 hours. Different magnetic field were generated and applied into the study material. The magnetic flux density was measured by a Hall effect sensor. The results were correlated with the ones of scanning electron microscopy (SEM), hardness and X-ray diffraction (XRD). The results showed that the Hall voltage measurements were able to follow the formation of $\sigma$ phase. The formation of this non-magnetic phase influenced the signal obtained by the Hall sensor, reducing the voltage. There was a correlation between magnetic flux density and the phase embrittlement, pointing to a methodology able to follow

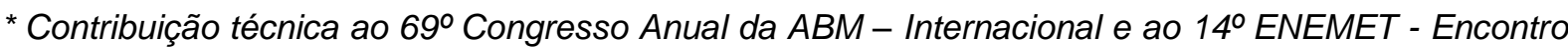
Nacional de Estudantes de Engenharia Metalúrgica, de Materiais e de Minas,21 a 25 de julho de 2014, São Paulo, SP, Brasil.
} 


\section{INTRODUÇÃO}

Métodos não destrutivos como os de velocidade e atenuação sônica, sinal retroespalhado de ultrassom, ruído Barkhausen e correntes parasitas, têm sido utilizados no estudo de transformações microestruturais [1-5]. O uso de materiais ferromagnéticos em aplicações da engenharia faz com que a perda de fluxo magnético seja uma das técnicas utilizadas para determinar a presença de descontinuidades tanto na superfície como também no seu interior [6].

Sensores Hall têm sido utilizados para detecção do tamanho e da posição de trincas em materiais. Esses sensores são sensíveis também à detecção de falhas superficiais em materiais metálicos, principalmente sob excitação de corrente alternada [7].

O efeito Hall, descoberto por Edwin Herbert Hall em 1879, já é conhecido por mais de cem anos, mas sua utilização se tornou viável nas últimas três décadas com o advento da microeletrônica. O efeito Hall é utilizado na indústria em uma ampla variedade de aplicações de sensoriamento, podendo ser encontrado em tacômetros, interruptores e medidores de posição, inclinação, nível, pressão, espessura, corrente, tensão, potência, frequência e campo magnético. Também pode ser encontrado na indústria de aviação, utilizado em avaliações não destrutivas [8].

O uso de propriedades magnéticas também já é bastante difundido na ciência dos materiais, mas até então tais propriedades foram utilizadas apenas em mecanismos de detecção de descontinuidades ou falhas [7].

Sensores de efeito Hall já foram utilizados para a caracterização microestrutural de aços submetidos a diversos tratamentos térmicos [9]. Amostras de aços carbono 1045 com diferentes tratamentos foram submetidas a um campo de 300 Gauss onde foram medidos valores de tensão Hall. Os resultados obtidos mostraram que o sensor Hall é sensível a mudança microestrutural, e que o mesmo, sendo utilizado para caracterização, se apresenta como um método não destrutivo promissor. Nesse trabalho, também foi mostrada a potencialidade da utilização de valores de tensão Hall associada a redes neurais artificiais no reconhecimento de microestruturas em um aço 1045 tratado termicamente. Sinais de tensão Hall serviram para treinar uma rede do tipo backpropagation, mostrando ser possível a identificação da microestrutura com a combinação das duas técnicas.

O aço inoxidável duplex tem como principais características a elevada resistência à corrosão e uma alta resistência mecânica, o que ocorre devido sua composição, formada, aproximadamente, por $50 \%$ de ferrita e $50 \%$ de austenita, sendo, por isso, considerado hoje tão importante o seu uso em indústrias do ramo petroquímico, alimentício, de papel e celulose e de petróleo e gás. Entretanto, quando aquecido a temperaturas superiores a $300^{\circ} \mathrm{C}$ esse tipo de aço perde sua tenacidade se tornando um material frágil devido ao surgimento de partículas ricas em cromo que empobrecem a matriz desse elemento [10].

As fases presentes nos aços inoxidáveis duplex submetidos a processamentos em altas temperaturas $(\sigma, \mathrm{X}, \mathrm{e} \delta)$ podem ser caracterizadas apenas através de métodos destrutivos tais como a microscopia eletrônica de varredura e a microscopia eletrônica de transmissão [11]. Técnicas não destrutivas tais como o ultrassom e as correntes parasitas têm sido estudadas para a detecção da fase $\sigma$ presente em aços inoxidáveis duplex em aplicações a altas temperaturas [2].

O estudo da fragilização do aço inoxidável duplex aplicado em componentes de usinas nucleares foi possível pela utilização da técnica não destrutiva de correntes parasitas. O método de correntes parasitas tem sido amplamente usado, pois possui

* Contribuição técnica ao 69 Congresso Anual da ABM - Internacional e ao 14ํㅡㄹ ENEMET - Encontro Nacional de Estudantes de Engenharia Metalúrgica, de Materiais e de Minas,21 a 25 de julho de 2014, São Paulo, SP, Brasil. 
equipamento leve e compacto e também permite testes sem contato através da corrente eletromagnética induzida. Esse método permite a quantificação da fragilização de aços inoxidáveis duplex através da comparação de resultados de materiais degradados com não degradados [3]. O estudo da precipitação da fase $\sigma$ pelo método de correntes parasitas, em amostras envelhecidas para tempos de até 2 horas nas temperaturas de 800 e $900{ }^{\circ} \mathrm{C}$ foi realizado e mostrou que a precipitação dessa fase pode ser acompanhada por medidas de impedância magnética [2].

O presente trabalho apresenta uma metodologia não destrutiva baseada na interação de linhas de campo magnético com o material de estudo. A formação da fase fragilizante $\sigma$ em um aço inoxidável duplex é acompanhada através dessa metodologia. Diferentes quantidades de fase $\sigma$ são produzidas através de um envelhecimento a $800{ }^{\circ} \mathrm{C}$ para tempos de até 2 horas. A formação dessa fase é analisada por medidas de tensão Hall.

\section{MATERIAIS E MÉTODOS}

Amostras de um aço inoxidável duplex UNS-S31803 foram envelhecidas na temperatura de $800^{\circ} \mathrm{C}$, com os seguintes tempos de envelhecimentos: $1 / 4,1$ e 2 horas, a fim de acompanhar a formação da fase $\sigma$. Estas e uma amostra na condição como recebida foram submetidas a ensaio de dureza Rockwell C, DRX, MEV e medidas de tensão utilizando sensores de efeito Hall. As três primeiras técnicas já são consolidadas e foram utilizadas para a comparação de seus resultados com os de medidas magnéticas da metodologia não destrutiva apresentada.

Os ensaios de DRX foram realizados no laboratório de solidificação rápida (LSR) do Centro de Tecnologia da Universidade Federal da Paraíba (CT/UFPB), com o objetivo de identificar as fases presentes no aço antes e após o tratamento térmico de envelhecimento. As amostras foram confeccionadas em formato retangular com comprimento, largura e espessura de 20,10 e $5 \mathrm{~mm}$, respectivamente, sendo uma amostra para cada tempo de envelhecimento. O equipamento utilizado foi um difratômetro SIEMENS D5000, operado segundo os parâmetros de radiação $k \alpha$ do Cobre, com tensão de $40 \mathrm{kV}$ e corrente de $30 \mathrm{~mA}$, passo de $0,02^{\circ}$, com tempo por passo igual a 9,6 segundos. Os ensaios foram realizados adotando um ângulo de varredura $(2 \theta)$ variando de 41 a $53^{\circ}$.

As superfícies das amostras tratadas foram preparadas por metalografia e atacadas para revelar a microestrutura do aço. Essas amostras foram submetidas a ataque químico pelo reagente Behara, em solução aquecida, composta de $20 \mathrm{ml}$ de ácido clorídrico $(\mathrm{HCl})$, adicionado em $100 \mathrm{ml}$ de água destilada e $0,5 \mathrm{~g}$ de metabissulfito de potássio $\left(\mathrm{K}_{2} \mathrm{~S}_{2} \mathrm{O}_{5}\right)$. A temperatura do banho variou entre 40 e $70^{\circ} \mathrm{C}$. As microestruturas foram analisadas em um microscópio eletrônico de varredura modelo 1430 da Oxford Instruments do Centro de Tecnologia do Gás (CTGAS/RN) e por microscópio ótico (marca Carl Zeiss) no Instituto Federal de Educação, Ciência e Tecnologia da Paraíba (IFPB).

Os ensaios de tensão Hall foram realizados no laboratório do Grupo de Simulação e Comportamento de Materiais (GSCMAT) do IFPB, utilizando uma configuração experimental constituída de um computador, uma placa de aquisição de dados, uma fonte de alimentação (CC), uma bancada de ensaios, um sensor de efeito Hall, uma amostra de aço inoxidável duplex e um eletroímã. Os campos magnéticos externos aplicados variaram entre 0 e 20 Oersted. A configuração experimental é apresentada na figura 1.

\footnotetext{
* Contribuição técnica ao $69^{\circ}$ Congresso Anual da ABM - Internacional e ao 14ํㅡㄹ ENEMET - Encontro Nacional de Estudantes de Engenharia Metalúrgica, de Materiais e de Minas,21 a 25 de julho de 2014, São Paulo, SP, Brasil.
} 

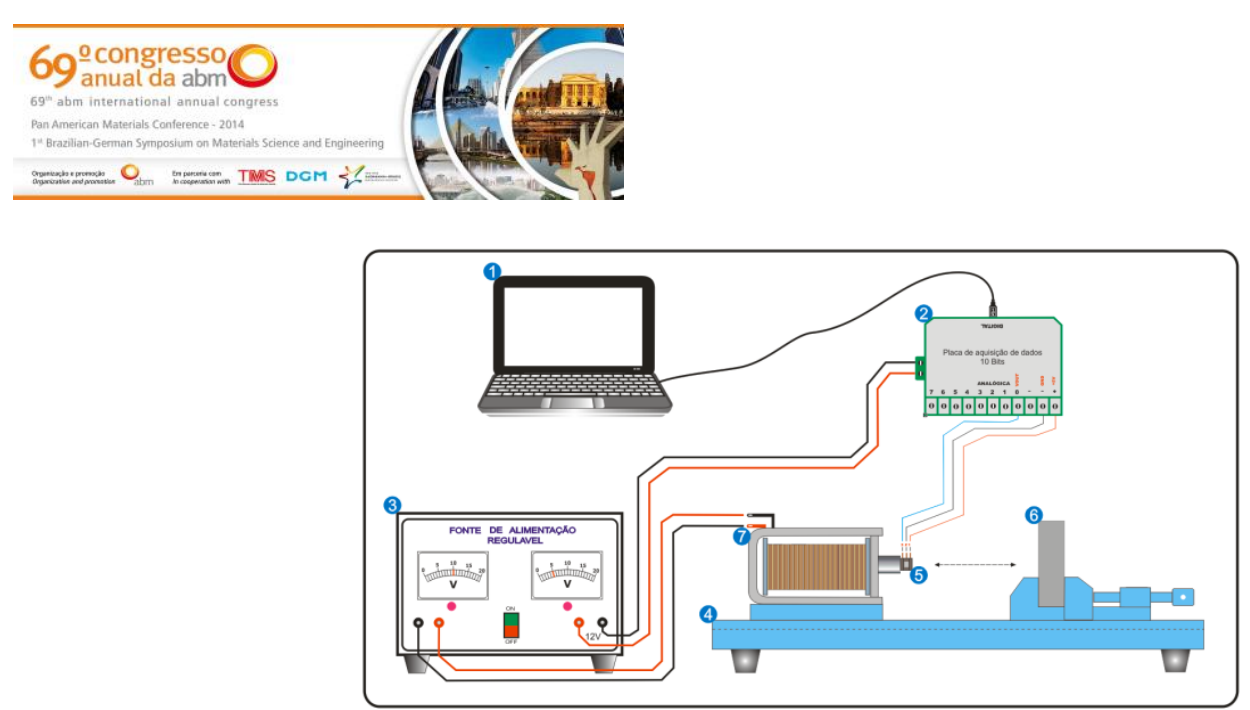

Figura 1. Configuração experimental do ensaio não destrutivo (END), contendo: (1) computador; (2) placa de aquisição de dados; (3) fonte de alimentação (CC); (4) bancada de ensaios; (5) sensor Hall; (6) amostra; (7) eletroímã.

O sensor utilizado é do tipo raciométrico linear de efeito Hall capaz de suportar operações em altas temperaturas, modelo A1323L, da Allegro Microsystems. Esse sensor fornece uma tensão de saída proporcional ao campo magnético aplicado.

\section{RESULTADOS E DISCUSSÃO}

A figura 2 mostra os difratogramas de raios $X$ e as microscopias eletrônicas de varredura do material como recebido e envelhecido a $800{ }^{\circ} \mathrm{C}$ por 1 e 2 horas. $\mathrm{Na}$ figura 2a, o material como recebido revela picos das fases austenita e ferrita. $\mathrm{Na}$ amostra envelhecida, figura $2 \mathrm{~b}$, nota-se a presença de um pico de pequena intensidade da fase $\sigma$. A figura 3c apresenta, além da fase $\sigma$, um pico da fase $X$ (essa última com pequena intensidade).

A fragilização do aço inoxidável duplex na faixa de temperatura de 600 a $1000{ }^{\circ} \mathrm{C}$ ocorre devido á presença da fase $\sigma$ [12]. Essa possui uma dureza em torno de 1000 HV e sua ocorrência em um porcentual de $4 \%$ já é suficiente para fragilizar o material e reduzir sua tenacidade em torno de $90 \%$. No presente trabalho foi observado um valor de $6,5 \%$ de fase $\sigma$ para a amostra envelhecida a $800^{\circ} \mathrm{C}$ por 2 horas e uma queda de tenacidade de $90 \%$. Essa medida de tenacidade foi determinada através de capacidade de absorção de energia por impacto Charpy e está de acordo com a literatura.

As figuras $2 \mathrm{~d}, 2 \mathrm{e}$ e $2 \mathrm{f}$ mostram as variações microestruturais para a amostra como recebida e as envelhecidas por 1 e 2 horas na temperatura de $800{ }^{\circ} \mathrm{C}$. Nota-se a formação de precipitados lamelares nas interfaces entre a austenita $(\gamma)$ e a ferrita (ठ), que cresce na direção da fase ferrítica. Isso pode ser melhor observado na microscopia da amostra envelhecida por 2 horas, apresentada na figura $2 f$. Essa formação ocorre através da reação eutetóide $(\delta \rightarrow \sigma+\gamma 2)$. A austenita secundária formada ( $\mathrm{\gamma} 2)$ também cresce da direção da fase ferrítica. Essa reação é atribuída ao fato de que durante a formação da fase $\sigma$, o cromo é absorvido e o níquel é rejeitado para regiões adjacentes dentro da fase ferrítica [13]. As setas nas figuras 2 e e $2 f$ indicam as estruturas lamelares da região que possui fase $\sigma$.

\footnotetext{
* Contribuição técnica ao $69^{\circ}$ Congresso Anual da ABM - Internacional e ao 14ํㅡㄹ ENEMET - Encontro Nacional de Estudantes de Engenharia Metalúrgica, de Materiais e de Minas,21 a 25 de julho de 2014, São Paulo, SP, Brasil.
} 


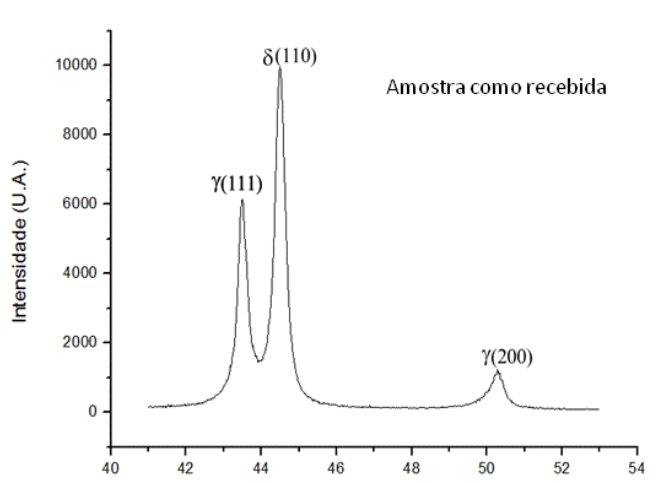

(a)

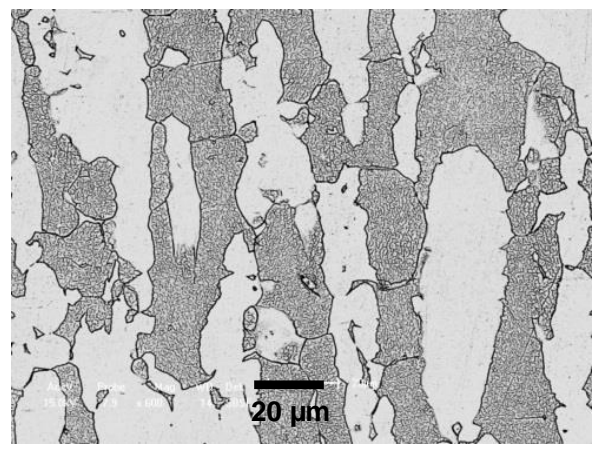

d)
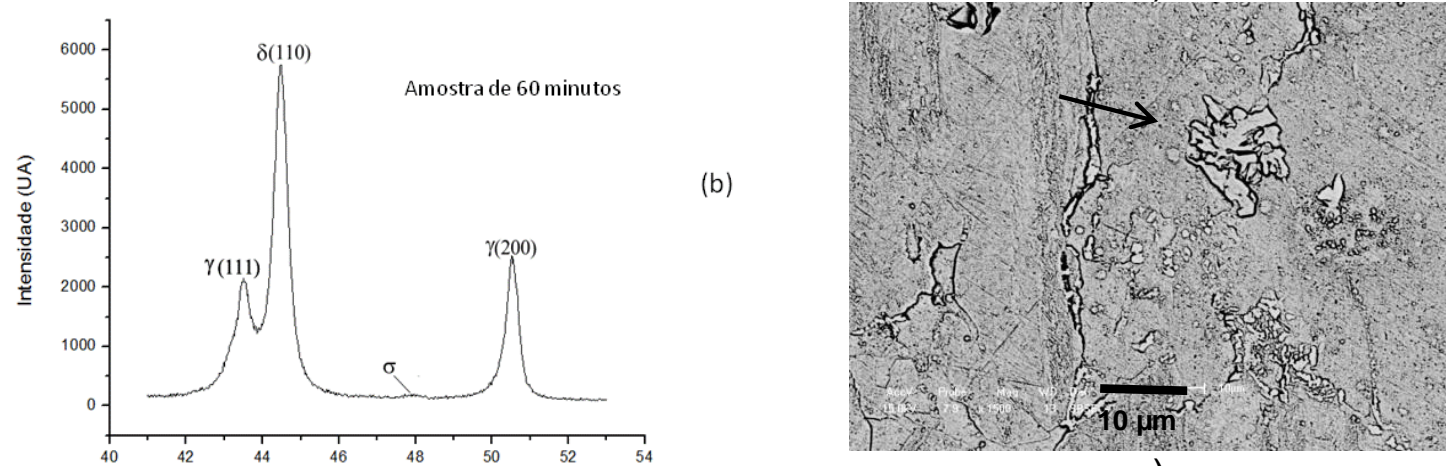

e)
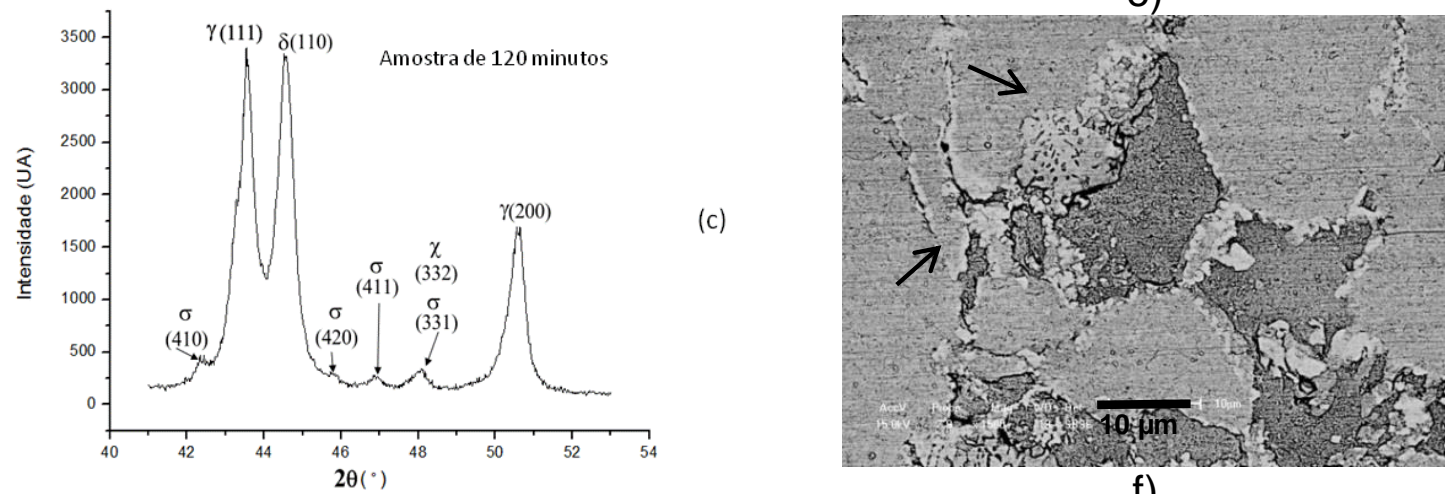

f)

Figura 2. a), b) e c) Difratograma de raios $X$ da amostra como recebida e das envelhecidas por $60 \mathrm{e}$ 120 min. d), e) e f) Microscopia eletrônica de varredura da amostra como recebida e das envelhecidas por 60 e 120 min.

A fase $\sigma$ presente nos aços inoxidáveis duplex pode ser caracterizada através de ensaios destrutivos como a análise por microscopia ótica, eletrônica de varredura e eletrônica de transmissão. Técnicas não destrutivas como o ultrassom e as correntes parasitas também têm sido usadas para detecção dessa fase em aços inoxidáveis duplex [2-4].

No presente trabalho foi avaliada a utilização de medidas de tensão Hall para acompanhamento da fase $\sigma$. $O$ valor de campo magnético externo ideal a ser aplicado foi obtido pela diferença entre os valores de campo induzido na amostra como recebida e na envelhecida por 2 horas (amplitude de medida), para diferentes campos externos aplicados. O campo externo correspondente à maior amplitude de medida foi denominado de campo ideal. Na figura 3 se tem a variação da amplitude calculada em função dos campos externos aplicados. O maior pico corresponde ao campo ideal para medição. O valor encontrado foi de 16 Oersted.

\footnotetext{
* Contribuição técnica ao 69ำ Congresso Anual da ABM - Internacional e ao 14ํㅡㄹ ENEMET - Encontro Nacional de Estudantes de Engenharia Metalúrgica, de Materiais e de Minas,21 a 25 de julho de 2014, São Paulo, SP, Brasil.
} 

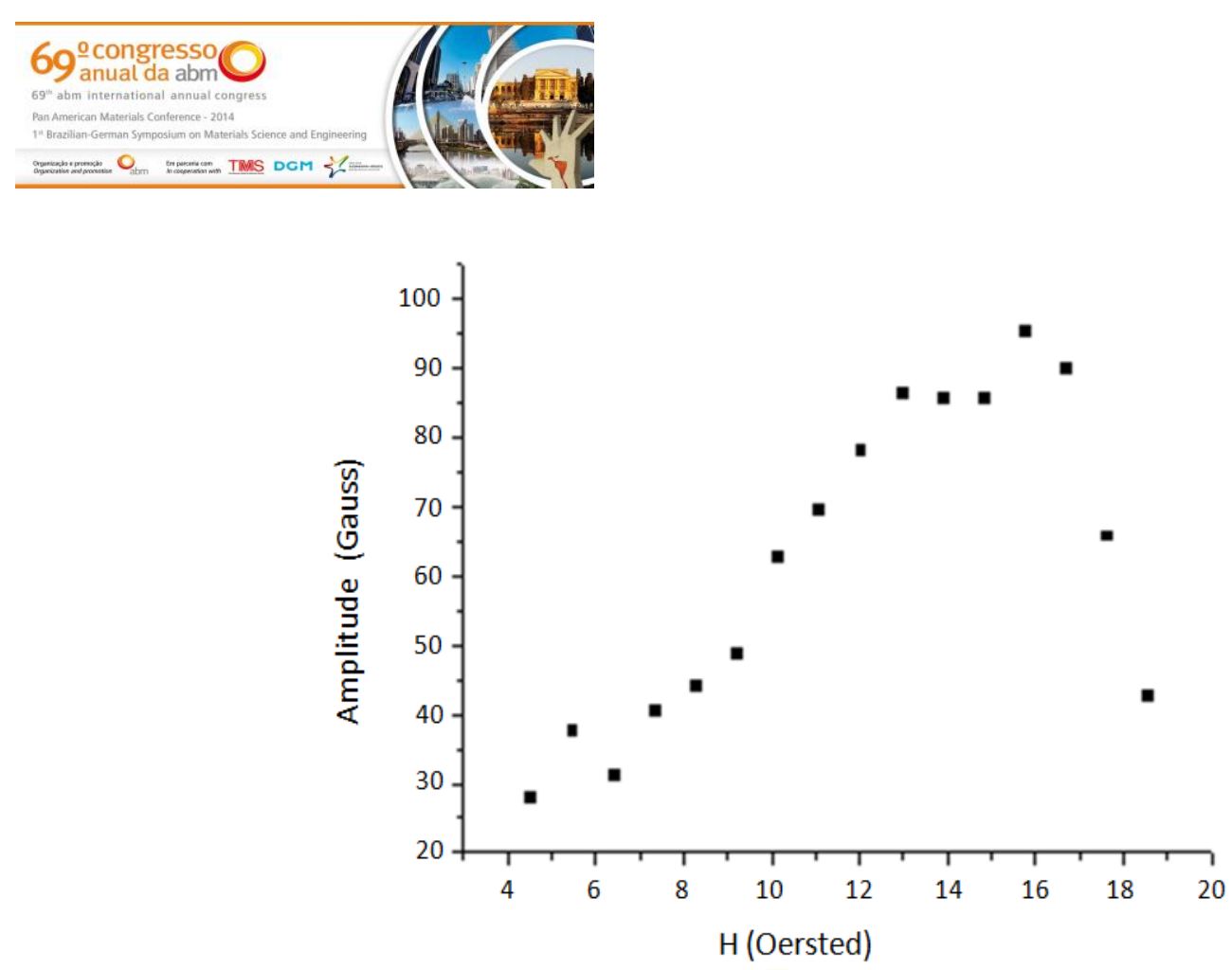

Figura 3. Amplitude calculada em função do campo externo aplicado.

Os valores de tensão Hall da amostra como recebida e das envelhecidas por 15, 60 e 120 minutos a $800^{\circ} \mathrm{C}$, para um campo externo de 16 Oersted são apresentados em função dos valores de dureza Rockwell $C$ na figura 4 . O rápido aumento de dureza está associado à formação da fase $\sigma$. A figura 4 mostra que os valores de dureza e tensão Hall são sensíveis à formação da fase $\sigma$. Essa fase, por não ser magnética, reduz os valores de tensão Hall. A formação de fases não magnéticas durante o envelhecimento altera a permeabilidade do meio e resulta na redução dos valores de tensão gerados pelo sensor de efeito Hall, que são proporcionais à densidade de linhas de campo do meio. Deste modo, o surgimento da fase $\sigma$ pode ser acompanhado por essa redução de valores e apontar para uma metodologia não destrutiva para monitoramento de estruturas que possam vir a falhar pela presença dessa fase.

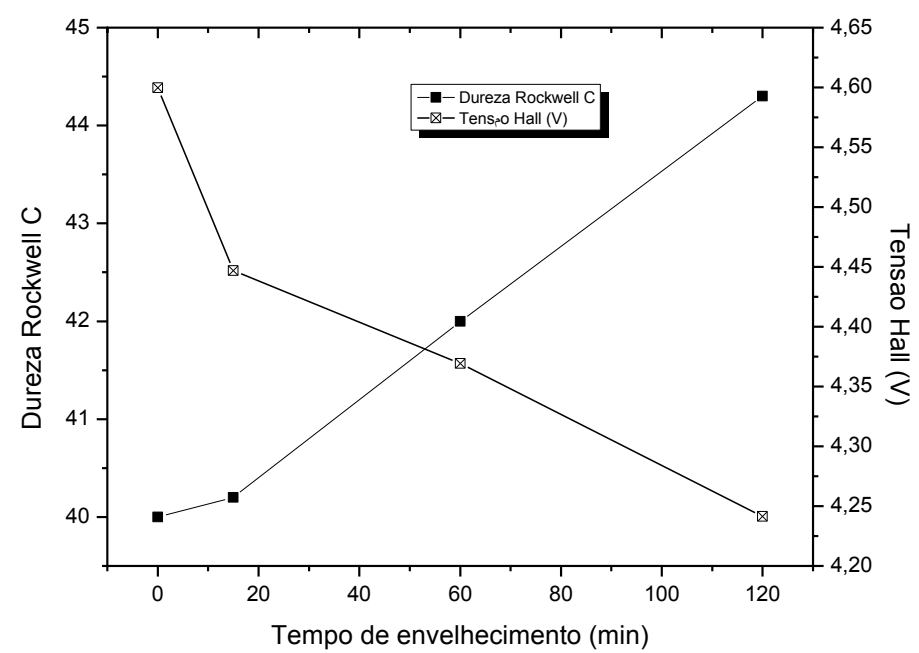

Figura 4. Variação das medidas de tensão Hall e dureza Rockwell C, em função do envelhecimento por até 2 horas a $800^{\circ} \mathrm{C}$.

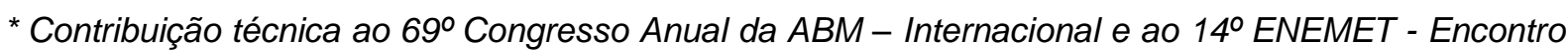
Nacional de Estudantes de Engenharia Metalúrgica, de Materiais e de Minas,21 a 25 de julho de 2014, São Paulo, SP, Brasil. 


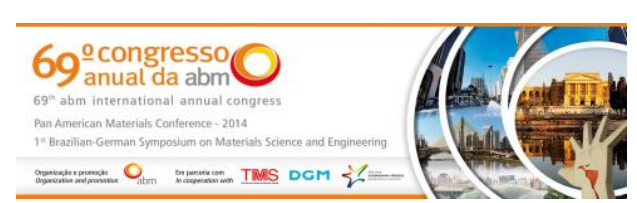

\section{CONCLUSÃo}

No presente trabalho foi estudada a possibilidade de acompanhamento da formação da fase $\sigma$ em um aço inoxidável duplex através da interação entre linhas de campo e microestrutura, chegando-se às seguintes conclusões:

- O aço inoxidável duplex apresenta na temperatura de estudo a formação da fase $\sigma$ através da decomposição da ferrita e a formação de duas fases não magnéticas, a fase $\sigma$ e a austenita secundária.

A metodologia apresentada é capaz de acompanhar a formação da fase $\sigma$, pois a formação de fases não magnéticas durante 0 envelhecimento altera a permeabilidade do meio e resulta na redução dos valores de tensão gerados pelo sensor de efeito Hall, que são proporcionais a densidades de linhas de campo do meio.

\section{Agradecimentos}

Os autores agradecem o suporte do IFPB para realização dos ensaios de Microscopia Eletrônica de Varredura e a Bolsa de pesquisador.

\section{REFERÊNCIAS}

1 Freitas VLA, Normando PG, Albuquerque VHC, Silva EM, Silva AA. Nondestructive characterization and evaluation of embrittlement kineties and elastic constants of duplex stainless steel SAF 2205 for different aging times at $425^{\circ} \mathrm{C}$ and $475^{\circ} \mathrm{C}$. Journal of Nondestructive Evatualion. 2011;30(3):130-136.

2 Normando PG, Moura EP, Souza JA, Tavares SSM, Padovese LR. Ultrasound, eddy current and magnetic Barkhausen noise as tools for sigma phase detection on a UNS S31803 duplex stainless steel. Mater. Sci. Eng.A. 2010;527:2886-2891.

3 Vijayalaksmi K, Muthpandi V, Jayachitra R. Influence of heat treatment on the microstructure, ultrasonic attenuation and hardness of SAF 2205 duplex stainless steel. Mater.Sci. Eng. A. 2011;529:447-451.

4 Tavares SSM, Pardal JM, Guerreiro JL, Gomes AM, Silva MR. Magnetic detection of sigma phase in duplex stainless steel UNS S31803. J. Magn. Magn. Mater. 2010;322:129-133.

5 Albuquerque VHC, Silva EM, Pereira LJ, Moura EP, Araújo VL, Tavares JMRS. Spinodal decomposition mechanism study on the duplex stainless steel UNS S31803 using ultrasonic speed measurements. Materials in Engineering, Materials and Design. 2010;31:2147-2150.

6 Enokizono M, Tokada T, Tuchida Y, Hil N. Finite element analysis of moving magnetic flux type sensor developed for nondestructive testing IEEE. Trans. Magn. 1999;35:18531956.

7 Bi Y, Jiles DC. Dependance of magnetic properties on crack size in steels, IEEE, Trans. Magn. 1998;34:2021-2224.

8 Kosmas K, Sargentis C, Tsamakis D, Hristoforou E. Non-destructive evaluation of magnetic metallic materials using Hall sensors. Journal of Materials Processing Technology. 2005;161:359-362.

9 Cavalcante AF, Albuquerque VHC, Silva EM, Santos NC, Silva AA. Um estudo da aplicação de um sensor Hall e uma rede neural artificial para reconhecimento dos diferentes tipos de tratamento térmico pelo qual um aço SAE 1045 foi submetido, IberianLatinAmerican Congresso on Computación Methods in Engineering - XXIX CILANCE, Maceio, Alagoas, Brazil, november 4th to 7th, 2008.

\footnotetext{
* Contribuição técnica ao $69^{\circ}$ Congresso Anual da ABM - Internacional e ao 14ํㅡㄹ ENEMET - Encontro Nacional de Estudantes de Engenharia Metalúrgica, de Materiais e de Minas,21 a 25 de julho de 2014, São Paulo, SP, Brasil.
} 
10 Silva EM, Albuquerque VHC, Leite JP, Varela ACG, Moura EP, Tavares JMRS. Phase Transformations Evaluation on an UNS S31803 Duplex Stainless Steel based on Nondestructive Testing. Materials Science \& Engineering. A, Structural Materials: properties, microstructure and processing, 2009; 516: 126-130.

11 Glosh SK, Mondal S. High temperature ageing behaviour of a duplex stainless steel, Materials Characterization, 2008; 59: 1776-1783.

12 Pardal JM, 2009, Efeitos dos Tratamentos Térmicos nas Propriedades Mecânicas, Magnéticas e na Resistência à Corrosão de Aços Inoxidáveis Supeduplex, Tese de D.Sc., 440 p., Depto. Eng. Mecânica/UFF, Rio de Janeiro, RJ, Brasil.

13 Romana R. Cinética de formação de fase sigma entre $700^{\circ} \mathrm{C}$ e $900^{\circ} \mathrm{C}$ no aço inoxidável superdúplex UNS S32750 (SAF 2507), Dissertação de Mestrado, FEI, São Bernardo do Campo, SP, Brasil, 2009.

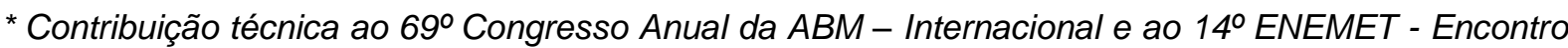
Nacional de Estudantes de Engenharia Metalúrgica, de Materiais e de Minas,21 a 25 de julho de 2014, São Paulo, SP, Brasil. 